\title{
THE NON-RELATIVISTIC LIMIT OF THE NORDSTRÖM-VLASOV SYSTEM*
}

\author{
SIMONE CALOGERO ${ }^{\dagger}$ AND HAYOUNG LEE A $^{\ddagger}$
}

\begin{abstract}
The Nordström-Vlasov system provides an interesting relativistic generalization of the Vlasov-Poisson system in the gravitational case, even though there is no direct physical application. The study of this model will probably lead to a better mathematical understanding of the class of non-linear systems consisting of hyperbolic and transport equations. In this paper it is shown that solutions of the Nordström-Vlasov system converge to solutions of the Vlasov-Poisson system in a pointwise sense as the speed of light tends to infinity, providing a further and rigorous justification of this model as a genuine relativistic generalization of the Vlasov-Poisson system.
\end{abstract}

\section{Introduction}

Kinetic models of collisionless matter have many important physical applications. In astrophysics, for example, the stars of a galaxy are often modelled as a large ensemble of particles in which collisions are sufficiently rare to be neglected. The distribution $f_{\infty}$ of particles in the phase-space satisfies the Vlasov-Poisson system:

$$
\begin{gathered}
\partial_{t} f_{\infty}+p \cdot \nabla_{x} f_{\infty}-\nabla_{x} U \cdot \nabla_{p} f_{\infty}=0, \\
\Delta_{x} U=4 \pi \gamma \rho_{\infty}, \quad \gamma=1, \quad \rho_{\infty}=\int_{\mathbb{R}^{3}} f_{\infty} d p .
\end{gathered}
$$

In the previous equations, $f_{\infty}=f_{\infty}(t, x, p)$ gives the probability density to find a particle (star) at time $t$ at position $x$ with momentum $p$, where $t \in \mathbb{R}, x \in \mathbb{R}^{3}, p \in \mathbb{R}^{3}$. $U=U(t, x)$ is the mean Newtonian potential generated by the stars.

By replacing $\gamma=-1$ in (1.2) one obtains the Vlasov-Poisson system in the plasma physics case. Here the particles are charges and $U$ is the electrostatic potential which they create collectively. We consider a single species of particle in both cases. The applications of these Vlasov-Poisson systems are restricted to the situations where the relativistic effects are negligible, i.e., low velocities and weak fields. Otherwise the dynamics has to be described by the relativistic Vlasov-Maxwell system in plasma physics and by the Einstein-Vlasov system in stellar dynamics.

The two Vlasov-Poisson models are very similar to each other and no substantial difference arises in the question of global existence of classical solutions, which is by now well-understood (cf. $[17,19,20,26]$ ). As opposed to this, the relativistic models have very different structure and so far they have been considered separately. In the gravitational case, global existence of (asymptotically flat) solutions for the EinsteinVlasov system is known only for small data with spherical symmetry [22]. For the relativistic Vlasov-Maxwell system the theory is more developed, cf. [3, 7], [9]-[15], [21]. However global existence and uniqueness of classical solutions for large data in three dimensions is still open.

In a recent paper [2], a different relativistic generalization to the Vlasov-Poisson system in the stellar dynamics case has been considered, in which the Vlasov dynamics is coupled to a relativistic scalar theory of gravity which goes back, essentially, to

\footnotetext{
${ }^{*}$ Received: September 12, 2003; accepted (in revised version): January 7, 2004. Communicated by Norbert Mauser.

†Department of Mathematics, Chalmers University, S-412 96 Göteborg, Sweden, (mg026@math.chalmers.se).

${ }^{\ddagger}$ Max-Planck-Institut für gravitationsphysik, Am Mühlenberg 1, D-14476 Golm (bei Potsdam), Germany, (hayoung@aei.mpg.de).
} 
Nordström [18]. More precisely, the gravitational theory considered in [2] corresponds to a reformulation of Nordström's theory due to Einstein and Fokker (see [8]). The resulting system has been called Nordström-Vlasov system and reads

$$
\begin{aligned}
& -\partial_{t}^{2} \phi+c^{2} \Delta_{x} \phi=4 \pi \int \frac{f d p}{\sqrt{1+c^{-2} p^{2}}}, \\
& \partial_{t} f+\widehat{p} \cdot \nabla_{x} f-\left[S(\phi) p+\frac{c^{2} \nabla_{x} \phi}{\sqrt{1+c^{-2} p^{2}}}\right] \cdot \nabla_{p} f=4 S(\phi) f,
\end{aligned}
$$

where

$$
p^{2}=|p|^{2}, \quad \widehat{p}=\left(1+c^{-2} p^{2}\right)^{-1 / 2} p, \quad S=\partial_{t}+\widehat{p} \cdot \nabla_{x} .
$$

Here $f=f(t, x, p), \phi=\phi(t, x)$ and $c$ denotes the speed of light. A solution $(f, \phi)$ of this system is interpreted as follows. The spacetime is a Lorentzian manifold with a conformally flat metric which, in the coordinates $(c t, x)$, takes the form

$$
g_{\mu \nu}=e^{2 \phi} \operatorname{diag}(-1,1,1,1) .
$$

Throughout the paper Greek indices $\mu, \nu$ and $\sigma$ run from 0 to 3 and Latin indices $a$ and $b$ take values $1,2,3$. The particle distribution $\tilde{f}$ defined on the mass shell in this metric is given by

$$
\tilde{f}(t, x, p)=e^{-4 \phi} f\left(t, x, e^{\phi} p\right) .
$$

More details on the derivation of this system are given in the next section. It should be emphasized that, although this model has no direct physical applications, scalar fields play a major role in modern theories of classical and quantum gravity. For example, the Brans-Dicke gravitational theory [1], which is continuously tested against general relativity, is a combination of Einstein's and Nordström's theory. The Nordström-Vlasov system is also interesting in a pure mathematical sense. A hope is that by studying this model one may reach a better understanding of a class of systems consisting of hyperbolic and transport equations.

However in order to justify this model as a genuine relativistic generalization of the (gravitational) Vlasov-Poisson system, it is necessary to indicate the relation between the solutions of the two systems. The main goal of this paper is to prove that in the non-relativistic limit $c \rightarrow \infty$ the solutions of (1.3)-(1.4) converge to solutions of (1.1)-(1.2) in a pointwise sense. The analogous result was proved in [25] for the relativistic Vlasov-Maxwell system (see [16] for the case of two space dimensions) and in [23] for the Einstein-Vlasov system with spherical symmetry (in the latter case a weaker form of convergence holds also in the absence of symmetries, see [24]).

This paper proceeds as follows. In section 2 we provide a formal derivation of the Nordström-Vlasov system and state our main results in full detail. The first of such results is a local existence theorem of solutions of the Nordström-Vlasov system in an interval of time independent of the speed of light, which is a necessary step to proceed further in the study of the non-relativistic limit. The solution of the latter problem is our second result. Our analysis follows [25] to a large extent and is based on the use of certain representation formulae for the solutions of the Nordström-Vlasov system which have been introduced in [4] and which will be adapted to the present case in section 3. There we shall also prove some estimates needed in the sequel. One of these estimates states that the distribution function $f$ is uniformly bounded, which 
permits it to improve the conditional global existence result, Theorem 1 in [4]. (The general $L^{q}$ estimates on $f$ are proved in [5], where they are used to establish existence of global weak solutions to the Nordström-Vlasov system). In section 4 we prove our main results.

\section{Derivation of the Nordström-Vlasov system and main results}

We shall refer to the Nordström-Vlasov system as the set of equations which models the kinetic motion of a self-gravitating ensemble of collisionless particles in accordance to a gravitational theory satisfying the following assumptions:

(1) The gravitational forces are mediated by a scalar field $\phi$ and the effect of such forces is to conformally rescale the metric of the (four dimensional) spacetime according to the relation

$$
g=A^{2}(\phi) \eta
$$

where $\eta$ is the Minkowski metric and $A$ is a positive function.

(2) Scale invariance property: There exists a one-parameter symmetry group whose action consists in rescaling $A(\phi)$ by a constant factor.

(3) Postulate of simplicity: The dynamics of the field $\phi$ is governed by second order differential equations.

(4) The matter (by which we mean any non-gravitational field) is universally coupled to the metric (2.1).

It was observed in [2] (appealing to the more general case of Scalar-Tensor theories considered in [6]) that the above assumptions single out a unique one-parameter family of scalar gravitation theories. This parameter appears because of the scale invariance property, which forces the conformal factor to be of the form $A(\phi)=\exp (\kappa \phi)$, with $\kappa>0$. Hence in this theory the spacetime is a Lorentzian manifold endowed with the metric

$$
g=e^{2 \kappa \phi} \eta
$$

To write down the field equation of this scalar gravitation theory in a simple form, let us consider a system of Cartesian coordinates $\left\{x^{0}:=c t, x^{1}, x^{2}, x^{3}\right\}=\left\{x^{\mu}\right\}, c$ denoting the vacuum speed of light in Galilean frames. In these coordinates, the equation for $\phi$ takes the form

$$
-c^{-2} \partial_{t}^{2} \phi+\Delta_{x} \phi=-4 \pi \frac{G_{*}}{c^{4}} \kappa e^{4 \kappa \phi} T .
$$

Here $G_{*}$ is a dimensional constant (the bare gravitational constant) and $T$ is the trace of the stress-energy tensor of the matter with respect to the physical metric $g$. In [2] $c, G_{*}$ and $\kappa$ have been set equal to unity and the factor $4 \pi$ has been removed for simplicity. As we already mentioned in the introduction, this scalar gravitation theory corresponds to the one considered in $[8,18]$.

In the case of the Nordström-Vlasov system, the dynamics of the matter is described by a non-negative, real-valued function $\tilde{f}$ which gives the probability density to find a particle in a given spacetime position $x^{\mu}$ and with a given four momentum $p^{\mu}$. We assume for simplicity that there is only one species of particle and choose units such that the proper mass of each particle is equal to one. The particle distribution $\tilde{f}$ is defined on the mass shell of the metric (2.2), which is the subset of the tangent bundle of spacetime defined by the condition $g_{\mu \nu} p^{\mu} p^{\nu}=-c^{2}, p^{0}>0$. This implies

$$
p^{0}=\sqrt{e^{-2 \kappa \phi} c^{2}+\delta_{a b} p^{a} p^{b}} .
$$


Using $\left(x^{\mu}, p^{a}\right)$ as coordinates on the mass shell and denoting by $d p$ the volume element $d p^{1} d p^{2} d p^{3}$, the stress-energy tensor for this matter model is

$$
T^{\mu \nu}=-c \int \sqrt{|\operatorname{det} g|} \frac{p^{\mu} p^{\nu}}{p_{0}} \tilde{f} d p,
$$

which implies

$$
T=-c^{3} e^{2 \kappa \phi} \int \frac{\tilde{f}}{p^{0}} d p .
$$

Finally, the coupling between the scalar gravitational field and the matter is completed by requiring that the distribution $\tilde{f}$ of particles on the mass-shell is constant on the geodesics of the metric (2.2). This leads to the Vlasov equation:

$$
c^{-1} \partial_{t} \tilde{f}+\frac{p^{a}}{p^{0}} \partial_{x^{a}} \tilde{f}-\frac{p^{\mu} p^{\nu}}{p^{0}} \Gamma_{\mu \nu}^{a} \partial_{p^{a}} \tilde{f}=0
$$

where $\Gamma_{\mu \nu}^{\sigma}=\kappa\left(\delta_{\nu}^{\sigma} \partial_{\mu} \phi+\delta_{\mu}^{\sigma} \partial_{\nu} \phi-\eta_{\mu \nu} \partial^{\sigma} \phi\right)$ are the Christoffel symbols of the metric $(2.2)$.

Our goal is to relate the solutions of the system $(2.3)-(2.6)$ to the solutions of the Vlasov-Poisson system (1.1)-(1.2) satisfying the condition $\lim _{|x| \rightarrow \infty} U=0$ (isolated solutions). Hence $\left(f_{\infty}, U\right)$ solves the system

$$
\begin{aligned}
& \partial_{t} f_{\infty}+p \cdot \nabla_{x} f_{\infty}-\nabla_{x} U \cdot \nabla_{p} f_{\infty}=0, \\
& U=-G \int \frac{\rho_{\infty}(t, y)}{|y-x|} d y \\
& \rho_{\infty}(t, x)=\int_{\mathbb{R}^{3}} f_{\infty}(t, x, p) d p
\end{aligned}
$$

where $G$ denotes the Newtonian gravitational constant which had been set equal to unity in (1.1)-(1.2). In order to get some light on the relation between the two systems, let us consider a formal expansion of the solutions of the Nordström-Vlasov system in powers of $1 / c$ :

$$
\begin{gathered}
\phi=\phi_{0}+c^{-1} \phi_{1}+c^{-2} \phi_{2}+\ldots \\
f=f_{0}+c^{-1} f_{1}+c^{-2} f_{2}+\ldots
\end{gathered}
$$

Substituting these into (2.3) and comparing the terms of the same order we obtain

$$
\begin{gathered}
\Delta_{x} \phi_{0}=0, \quad \Delta_{x} \phi_{1}=0 \\
-\partial_{t}^{2} \phi_{0}+\Delta_{x} \phi_{2}=4 \pi G_{*} \kappa e^{7 \kappa \phi_{0}} \int f_{0} d p .
\end{gathered}
$$

Assuming fields vanishing at infinity, (2.10) implies $\phi_{0}=\phi_{1}=0$ and so (2.11) reduces to (2.8) with the identification $\phi_{2} \sim U, f_{0} \sim f_{\infty}$, provided that $G_{*} \kappa=G$. The latter condition, which is necessary in order to obtain the correct Newtonian limit, shows that the role of the scale invariance parameter $\kappa$ is merely the one of fixing the units of the corresponding theory. We shall henceforth set $\kappa=G_{*}=G=1$ for simplicity.

To put the above formal discussion in a more rigorous mathematical context, we first rewrite the equations (2.3)-(2.6) with the "unphysical" particle density as in the formulation of [4], namely

$$
f(t, x, p)=e^{4 \phi} \tilde{f}\left(t, x, e^{-\phi} p\right) .
$$


In this frame, the unknown $(f, \phi)$ satisfies the equations (1.3)-(1.4). We supply this system with initial data $0 \leq f(0, x, p)=f^{\text {in }}(x, p), \phi(0, x)=\phi_{0}^{\text {in }}(x), \partial_{t} \phi(0, x)=\phi_{1}^{\text {in }}(x)$.

The following notation will be used. Given two functions $g$ and $h$ on $\mathbb{R}^{n}$ we write $g \lesssim h$ if the estimate $g \leq D h$ holds for a non-negative constant $D$ independent of $c \geq 1$. The constant $D$ may also depend on the length of some time interval $[0, T]$, in which case we write $g \lesssim h$ for $t \in[0, T]$. Furthermore we write

$$
A=B+\mathcal{O}\left(c^{-\delta}\right), \quad \delta \geq 1
$$

if $|A(y)-B(y)| \lesssim c^{-\delta}, \forall y \in \mathbb{R}^{n}$. We also set

$$
\mathcal{P}_{c}(t)=\sup _{0 \leq s<t}\{|p|:(x, p) \in \operatorname{supp} f(s)\}+1
$$

where supp $f(t)$ means the support of $f(t, x, p)$ on $(x, p) \in \mathbb{R}^{6}$ for each $t$.

Here are the main results of this paper:

THEOREM 2.1. Initial data $f^{\text {in }} \in C_{c}^{1}\left(\mathbb{R}^{6}\right), \phi_{0}^{\text {in }} \in C_{b}^{3}\left(\mathbb{R}^{3}\right), \phi_{1}^{\text {in }} \in C_{b}^{2}\left(\mathbb{R}^{3}\right)$ launch a unique classical solution $(f, \phi) \in C^{1}\left(\left[0, T_{\max }\right) \times \mathbb{R}^{6}\right) \times C^{2}\left(\left[0, T_{\max }\right) \times \mathbb{R}^{3}\right)$ to the Cauchy problem for the Nordström-Vlasov system (1.3)-(1.4) in a maximal interval of time $\left[0, T_{\max }\right)$. If $\mathcal{P}_{c}\left(T_{\max }\right)<\infty$, then $T_{\max }=\infty$, i.e., the solution is global.

Note that under the assumptions of Theorem 2.1 the local time of existence may shrink to zero as the speed of light tends to $+\infty$. To remove this possibility we specify more restrictive initial data:

ThEOREM 2.2. Assume $f^{\text {in }} \in C_{c}^{3}\left(\mathbb{R}^{6}\right)$ and $\phi_{0}^{\text {in }}=c^{-2} g^{\sharp}, \phi_{1}^{\text {in }}=c^{-2} h^{\sharp}$, where

$$
g^{\sharp}(x)=-\iint \frac{f^{\text {in }}(y, p)}{|y-x|} d p d y, \quad h^{\sharp} \in C_{c}^{2}\left(\mathbb{R}^{3}\right) .
$$

Corresponding to these data there exists a unique solution $(f, \phi) \in C^{1}\left([0, T) \times \mathbb{R}^{6}\right) \times$ $C^{2}\left([0, T) \times \mathbb{R}^{3}\right)$ of (1.3)-(1.4) in an interval of time $[0, T)$ independent of $c$ such that $\mathcal{P}_{c}(T) \lesssim 1$.

Finally we give the conditions under which solutions to the Nordström-Vlasov system converge in a pointwise sense to solutions of the Vlasov-Poisson system in the non-relativistic limit.

THEOREM 2.3. Let the data for the Nordström-Vlasov system be given as in Theorem 2.2 and assume that

(*) There exists a unique solution $(f, \phi) \in C^{1}\left([0, T) \times \mathbb{R}^{6}\right) \times C^{2}\left([0, T) \times \mathbb{R}^{3}\right)$ of (1.3)-(1.4) in an interval $[0, T) \subseteq[0,+\infty)$ independent of $c$ and the estimate $\mathcal{P}_{c}\left(T^{\prime}\right) \lesssim 1$ holds for all $0 \leq T^{\prime}<T$.

Denote by $f_{\infty} \in C^{1}\left([0, \infty) \times \mathbb{R}^{6}\right)$ the global solution of (2.7)-(2.9) with data $f^{\text {in }}$, which is known to exist by [19]. Then for every $T^{\prime} \in[0, T)$ and $t \in\left[0, T^{\prime}\right]$ :

$$
\begin{gathered}
\partial_{t} \phi(t)=\mathcal{O}\left(c^{-1}\right), \quad c^{2} \phi(t)=U(t)+\mathcal{O}\left(c^{-1}\right), \quad c^{2} \nabla_{x} \phi(t)=\nabla_{x} U(t)+\mathcal{O}\left(c^{-1}\right), \\
f(t)=f_{\infty}(t)+\mathcal{O}\left(c^{-1}\right)
\end{gathered}
$$

In the notation of the spaces of functions used above, the subscript $c$ indicates that functions are compactly supported and $b$ means that all the derivatives up to the 
indicated order are bounded. Theorem 2.1 will be proved as a corollary of Theorem 1 of [4] in the next section and Theorems 2.2 and 2.3 will be proved in Section 4.

To conclude this section, we remark that more general data for the field are allowed in Theorems 2.2 and 2.3. For instance we may require that $\phi_{0}^{\text {in }}=c^{-2} g^{\sharp}+c^{-3} g^{b}$, where $g^{b} \in C_{b}^{3}\left(\mathbb{R}^{3}\right)$. This modifies our estimates only by terms which are of higher order in powers of $c^{-1}$, without affecting the general argument. In order to make the estimates below more transparent, we take for simplicity $g^{b} \equiv 0$.

\section{Preliminaries and proof of Theorem 2.1}

First note that the classical solution of (1.3) is

$$
\begin{aligned}
\phi(t, x) & =\phi_{\mathrm{hom}}(t, x)-\frac{1}{c^{2}} \int_{|y-x| \leq c t} \int \frac{f\left(t-c^{-1}|y-x|, y, p\right)}{\sqrt{1+c^{-2} p^{2}}|y-x|} d p d y \\
& :=\phi_{\mathrm{hom}}(t, x)+\psi(t, x),
\end{aligned}
$$

where

$$
\phi_{\text {hom }}(t, x)=\partial_{t}\left(\frac{t}{4 \pi} \int_{|\omega|=1} \phi_{0}^{\text {in }}(x+c t \omega) d \omega\right)+\frac{t}{4 \pi} \int_{|\omega|=1} \phi_{1}^{\text {in }}(x+c t \omega) d \omega
$$

is the solution of the homogeneous wave equation with data $\phi_{0}^{\text {in }}$ and $\phi_{1}^{\text {in }}$ and $\psi$ the solution of (1.3) with trivial data. We start with estimating the homogeneous part of the field $\phi$.

Lemma 3.1. Let the initial data for the field be given as in Theorems 2.2 and 2.3. Then we have

$$
\left\|\phi_{\text {hom }}(t)\right\|_{\infty} \lesssim c^{-1}(1+t)
$$

Proof: By means of (3.2) and the assumptions on the data $\phi_{0}^{\text {in }}$ and $\phi_{1}^{\text {in }}$ we have

$$
\phi_{\text {hom }}\left(c^{-1} t, x\right)=\frac{1}{c^{2}}\left[\partial_{t}\left(\frac{t}{4 \pi} \int_{|\omega|=1} g^{\sharp}(x+t \omega) d \omega\right)+\frac{c^{-1} t}{4 \pi} \int_{|\omega|=1} h^{\sharp}(x+t \omega) d \omega\right] .
$$

The term in the square brackets in (3.3) is estimated by $D(1+t)$ where

$$
D:=\left\|g^{\sharp}\right\|_{\infty}+\left\|\nabla_{x} g^{\sharp}\right\|_{\infty}+\left\|h^{\sharp}\right\|_{\infty} .
$$

Hence $\left\|\phi_{\text {hom }}\left(c^{-1} t\right)\right\|_{\infty} \lesssim c^{-2}(1+t)$, which implies $\left\|\phi_{\text {hom }}(t)\right\|_{\infty} \lesssim c^{-1}(1+t)$.

The following estimate is crucial for extending the argument of [25] to the Nordström-Vlasov system.

Proposition 3.2. The distribution function $f$ satisfies the estimate

$$
\|f(t)\|_{\infty} \leq\left\|f^{\mathrm{in}}\right\|_{\infty} \exp \left[4\left(\left\|\phi_{\text {hom }}(t)\right\|_{\infty}+\left\|\phi_{0}^{\mathrm{in}}\right\|_{\infty}\right)\right] .
$$

In particular, for data as in Theorems 2.2 and 2.3, we have $\|f(t)\|_{\infty} \lesssim e^{4 D t}$ for all $t \in \mathbb{R}$.

Proof : Let $(X, P)(s, t, x, p)$ denote the characteristics of (1.4) which satisfy the condition $(X, P)(t, t, x, p)=(x, p)$. In short, we use $X(s):=X(s, t, x, p)$ and $P(s):=$ 
$P(s, t, x, p)$ instead. Note that the function $e^{-4 \phi} f$ is constant along these curves. Hence the solution of (1.4) is given by

$$
\begin{aligned}
f(t, x, p) & =f^{\text {in }}(X(0), P(0)) \exp \left[4 \phi(t, x)-4 \phi^{\text {in }}(X(0))\right] \\
& =f^{\text {in }}(X(0), P(0)) \exp \left[-4 \phi^{\text {in }}(X(0))\right] \exp \left[4 \phi_{\text {hom }}(t, x)\right] \exp [4 \psi(t, x)] .
\end{aligned}
$$

Since $\psi \leq 0$, then $e^{4 \psi} \leq 1$ and the claim follows.

Combining this result with the one in [4] we obtain the following.

Proof of Theorem 2.1: It is enough to prove the theorem for $c=1$. For given a solution $(f, \phi)$ of (1.3)-(1.4), then the solution obtained by the rescaling $c f\left(c^{-1} t, x, c p\right)$, $\phi\left(c^{-1} t, x\right)$ solves the system with $c=1$. The claim has been proved in [4] under the additional condition that $\mathcal{Q}\left(T_{\max }\right)<\infty$, where

$$
\mathcal{Q}(t)=\sup _{0 \leq s<t}\{|\phi(t, x)|:(x, p) \in \operatorname{supp} f(s)\} .
$$

Now, assuming compact support in $p$ for the distribution function, i.e., $\mathcal{P}_{1}\left(T_{\max }\right)<\infty$, it follows by Proposition 3.2 that the right hand side of (3.1) is bounded in $L^{\infty}$. Hence $\phi$ itself is bounded and thus the condition $\mathcal{Q}\left(T_{\max }\right)<\infty$ is satisfied. The claim follows by Theorem 1 of [4].

Next we derive the representation formulae for the first order derivatives of the field as in [4], but for arbitrary values of $c$ and also with all data terms specified.

One can see by the Vlasov equation (1.4) that

$$
S f=\left\{\left(\partial_{t} \phi\right) p+c^{2}\left(1+c^{-2} p^{2}\right)^{-1 / 2} \nabla_{x} \phi\right\} \cdot \nabla_{p} f+4(S \phi) f .
$$

From (3.1) we have

$$
\begin{aligned}
\partial_{t} \phi(t, x)= & \partial_{t} \phi_{\text {hom }}(t, x)-c^{-2} t^{-1} \int_{|y-x|=c t} \int \frac{f^{\text {in }}(y, p)}{\sqrt{1+c^{-2} p^{2}}} d p d S_{y} \\
& -c^{-2} \int_{|y-x| \leq c t} \int \frac{\partial_{t} f\left(t-c^{-1}|y-x|, y, p\right)}{\sqrt{1+c^{-2} p^{2}}|y-x|} d p d y .
\end{aligned}
$$

Now using the identity

$$
\begin{aligned}
\partial_{t} g\left(t-c^{-1}|y-x|, y, p\right)= & \left(1+c^{-1} \omega \cdot \widehat{p}\right)^{-1}\left\{(S g)\left(t-c^{-1}|y-x|, y, p\right)\right. \\
& \left.-\widehat{p} \cdot \nabla_{y}\left[g\left(t-c^{-1}|y-x|, y, p\right)\right]\right\}
\end{aligned}
$$

and integration by parts we achieve the following representation for $\partial_{t} \phi$.

Proposition 3.3.

$$
\begin{aligned}
\partial_{t} \phi(t, x) & =\partial_{t} \phi_{\mathrm{hom}}(t, x) \\
& -c^{-2} t^{-1} \int_{|y-x|=c t} \int \frac{f^{\mathrm{in}}(y, p)}{\left(1+c^{-1} \omega \cdot \widehat{p}\right) \sqrt{1+c^{-2} p^{2}}} d p d S_{y} \\
& -c^{-2} \int_{|y-x| \leq c t} \int a^{\phi_{t}}(\omega, p) f\left(t-c^{-1}|y-x|, y, p\right) d p \frac{d y}{|y-x|^{2}} \\
& -c^{-2} \int_{|y-x| \leq c t} \int b^{\phi_{t}}(\omega, p)(S \phi) f\left(t-c^{-1}|y-x|, y, p\right) d p \frac{d y}{|y-x|} \\
& -c^{-1} \int_{|y-x| \leq c t} \int c^{\phi_{t}}(\omega, p)\left(\nabla_{x} \phi\right) f\left(t-c^{-1}|y-x|, y, p\right) d p \frac{d y}{|y-x|},
\end{aligned}
$$


where the kernels are

$$
\begin{aligned}
a^{\phi_{t}}(\omega, p) & =-\frac{\widehat{p} \cdot\left(\omega+c^{-1} \widehat{p}\right)}{\left(1+c^{-1} \omega \cdot \widehat{p}\right)^{2} \sqrt{1+c^{-2} p^{2}}} \\
b^{\phi_{t}}(\omega, p) & =\frac{\left(\omega+c^{-1} \widehat{p}\right)^{2}}{\left(1+c^{-1} \omega \cdot \widehat{p}\right)^{2} \sqrt{1+c^{-2} p^{2}}} \\
c^{\phi_{t}}(\omega, p) & =\frac{\omega+c^{-1} \widehat{p}}{\left(1+c^{-1} \omega \cdot \widehat{p}\right)^{2}\left(1+c^{-2} p^{2}\right)^{3 / 2}}
\end{aligned}
$$

and $\omega=(y-x) /|y-x|$.

The process to obtain the representation for $\nabla_{x} \phi$ is similar to the way for $\partial_{t} \phi$, but now with the following identity:

$$
\begin{aligned}
\partial_{y_{i}} g\left(t-c^{-1}|y-x|, y, p\right) & =c^{-1} \omega_{i}\left(1+c^{-1} \omega \cdot \widehat{p}\right)^{-1}(S g)\left(t-c^{-1}|y-x|, y, p\right) \\
& +\left(\delta_{i k}-\frac{c^{-1} \omega_{i} \widehat{p}_{k}}{1+c^{-1} \omega \cdot \widehat{p}}\right) \partial_{y_{k}}\left[g\left(t-c^{-1}|y-x|, y, p\right)\right], i=1,2,3
\end{aligned}
$$

Proposition 3.4. The representation of $\partial_{x_{i}} \phi$ is

$$
\begin{aligned}
& \partial_{x_{i}} \phi(t, x)=\partial_{x_{i}} \phi_{\text {hom }}(t, x) \\
& -c^{-3} t^{-1} \int_{|y-x|=c t} \int \frac{\omega_{i}}{\left(1+c^{-1} \omega \cdot \widehat{p}\right) \sqrt{1+c^{-2} p^{2}}} f^{\text {in }}(y, p) d p d S_{y} \\
& -c^{-3} \int_{|y-x| \leq c t} \int a^{\phi_{x_{i}}}(\omega, p) f\left(t-c^{-1}|y-x|, y, p\right) d p \frac{d y}{|y-x|^{2}} \\
& -c^{-3} \int_{|y-x| \leq c t} \int b^{\phi_{x_{i}}}(\omega, p)(S \phi) f\left(t-c^{-1}|y-x|, y, p\right) d p \frac{d y}{|y-x|} \\
& -c^{-2} \int_{|y-x| \leq c t} \int c^{\phi_{x_{i}}}(\omega, p)\left(\nabla_{x} \phi\right) f\left(t-c^{-1}|y-x|, y, p\right) d p \frac{d y}{|y-x|}
\end{aligned}
$$

where the kernels are

$$
\begin{aligned}
& a^{\phi_{x_{i}}}(\omega, p)=\frac{c\left(\omega+c^{-1} \widehat{p}\right)_{i}-c^{-1}(\widehat{p} \wedge(\omega \wedge \widehat{p}))_{i}}{\left(1+c^{-1} \omega \cdot \widehat{p}\right)^{2} \sqrt{1+c^{-2} p^{2}}} \\
& b^{\phi_{x_{i}}}(\omega, p)=\omega_{i} b^{\phi_{t}} \\
& c^{\phi_{x_{i}}}(\omega, p)=\omega_{i} c^{\phi_{t}} .
\end{aligned}
$$

\section{Proof of Theorems 2.2 and 2.3}

In this section we prove our main results. We shall frequently use Lemmas 1 and 2 of [25], which we state below for future reference.

Lemma 4.1. For all $g \in C_{c}^{0}\left(\mathbb{R}^{3}\right)$, we have

$$
\xi \int_{|\omega|=1}|g(x+\xi \omega)| d \omega \lesssim 1
$$

for $\xi \geq 0$. 
Lemma 4.2. Let $h \in C^{2}\left(\mathbb{R}^{3}\right)$ such that $\Delta h \in C_{c}^{0}\left(\mathbb{R}^{3}\right)$. Then for $c>0$ and $t \geq 0$,

$$
\partial_{t}\left(t \int_{|\omega|=1} h(x+c t \omega) d \omega\right)=-\int_{|y-x|>c t} \frac{\Delta h(y)}{|y-x|} d y .
$$

The next Lemma contains two simple estimates which are often used in the sequel.

LEMMA 4.3.

(i) $\left(1+c^{-1} \omega \cdot \widehat{p}\right)^{-1} \lesssim \mathcal{P}_{c}(t)^{2}$, for $(x, p) \in \operatorname{supp} f(t)$

(ii) $f(t, x, p)=0$, for $|x| \geq R+\mathcal{P}_{c}(t) t$, where $R:=\sup \left\{|x|:(x, p) \in \operatorname{supp} f^{\text {in }}\right\}$.

Proof: $\mathrm{By}|p| \leq \mathcal{P}_{c}(t)$,

$$
1+c^{-1} \omega \cdot \widehat{p} \geq 1-\frac{|p|}{\sqrt{c^{2}+p^{2}}}=\frac{c^{2}}{\sqrt{c^{2}+p^{2}}\left(\sqrt{c^{2}+p^{2}}+|p|\right)} \geq \frac{c^{2}}{2\left(c^{2}+\mathcal{P}_{c}(t)^{2}\right)},
$$

by which (i) follows. The property (ii) on the support of $f$ follows by (3.4) and the definition of characteristics.

Proof of Theorem 2.2: From Proposition 3.4, we have

$$
\begin{aligned}
& \partial_{x_{i}} \phi(t, x)=\partial_{x_{i}} \phi_{\mathrm{hom}}(t, x) \\
& \quad-c^{-3} t^{-1} \int_{|y-x|=c t} \int \frac{\omega_{i}}{\left(1+c^{-1} \omega \cdot \widehat{p}\right) \sqrt{1+c^{-2} p^{2}}} f^{\mathrm{in}}(y, p) d p d S_{y} \\
& \quad+I_{x_{i}}+I I_{x_{i}}+I I I_{x_{i}},
\end{aligned}
$$

where

$\partial_{x_{i}} \phi_{\text {hom }}(t, x)=c^{-2} \partial_{t}\left(\frac{t}{4 \pi} \int_{|\omega|=1} \partial_{x_{i}} g^{\sharp}(x+c t \omega) d \omega\right)+c^{-2} \frac{t}{4 \pi} \int_{|\omega|=1} \partial_{x_{i}} h^{\sharp}(x+c t \omega) d \omega$.

By the assumption $h^{\sharp} \in C_{c}^{2}\left(\mathbb{R}^{3}\right)$, Lemma 4.1 gives

$$
\left|\frac{c^{-2} t}{4 \pi} \int_{|\omega|=1} \partial_{x_{i}} h^{\sharp}(x+c t \omega) d \omega\right| \lesssim c^{-3} .
$$

For the first term in (4.2), using Lemma 4.2 and the identity $\Delta g^{\sharp}=4 \pi \int f^{\text {in }} d p$ we get

$$
\begin{aligned}
\partial_{t}\left(\frac{t}{4 \pi} \int_{|\omega|=1} \partial_{x_{i}} g^{\sharp}(x+c t \omega) d \omega\right)= & -\partial_{x_{i}} \int_{|y-x|>c t} \int \frac{f^{\mathrm{in}}(y, p)}{|y-x|} d p d y \\
=(c t)^{-1} \int_{|y-x|=c t} \int \omega_{i} f^{\mathrm{in}}(y, p) d p d S_{y} & -\int_{|y-x|>c t} \int \frac{\omega_{i} f^{\mathrm{in}}(y, p)}{|y-x|^{2}} d p d y .
\end{aligned}
$$

The surface integral will be combined with the second term in (4.1). First note that, 
using (i) in Lemma 4.3 and $|\widehat{p}| \leq \mathcal{P}_{c}(t)$,

$$
\begin{aligned}
\mid 1 & -\frac{1}{\left(1+c^{-1} \omega \cdot \widehat{p}\right) \sqrt{1+c^{-2} p^{2}}} \mid \\
& \leq\left|1-\frac{1}{\sqrt{1+c^{-2} p^{2}}}\right|+\left|\frac{1}{\sqrt{1+c^{-2} p^{2}}}-\frac{1}{\left(1+c^{-1} \omega \cdot \widehat{p}\right) \sqrt{1+c^{-2} p^{2}}}\right| \\
& \lesssim c^{-2} \mathcal{P}_{c}(t)^{2}+\frac{1}{\sqrt{1+c^{-2} p^{2}}}\left[\frac{c^{-1}|\omega \cdot \widehat{p}|}{\left(1+c^{-1} \omega \cdot \widehat{p}\right)}\right] \lesssim c^{-1} \mathcal{P}_{c}(t)^{3} .
\end{aligned}
$$

So we get

$$
\begin{aligned}
& \mid c^{-3} t^{-1} \int_{|y-x|=c t} \int \omega_{i} f^{\text {in }}(y, p) d p d S_{y} \\
& -c^{-3} t^{-1} \int_{|y-x|=c t} \int \frac{\omega_{i} f^{\text {in }}(y, p) d p d S_{y}}{\left(1+c^{-1} \omega \cdot \widehat{p}\right) \sqrt{1+c^{-2} p^{2}}} \mid \\
& \quad \lesssim c^{-3} t^{-1} \int_{|y-x|=c t} \int c^{-1} \mathcal{P}_{c}(t)^{3}\left|\omega_{i}\right| f^{\text {in }}(y, p) d p d S_{y} \\
& \quad \lesssim c^{-3} \mathcal{P}_{c}(t)^{3}\left(c t \int_{|\omega|=1} \int f^{\text {in }}(x+c t \omega, p) d p d \omega\right) \lesssim c^{-3} \mathcal{P}_{c}(t)^{3} .
\end{aligned}
$$

Split the kernel in $I_{x_{i}}$ according to

$$
\tilde{a}^{\phi_{x_{i}}}(\omega, p):=\frac{\widehat{p}_{i}-c^{-1}(\widehat{p} \wedge(\omega \wedge \widehat{p}))_{i}}{\left(1+c^{-1} \omega \cdot \widehat{p}\right)^{2} \sqrt{1+c^{-2} p^{2}}}=a^{\phi_{x_{i}}}(\omega, p)-\frac{c \omega_{i}}{\left(1+c^{-1} \omega \cdot \widehat{p}\right)^{2} \sqrt{1+c^{-2} p^{2}}} .
$$

By (i) in Lemma 4.3 one can see that $\left|\tilde{a}^{\phi_{x_{i}}}\right| \lesssim \mathcal{P}_{c}(t)^{5}$. Using Proposition 3.2 and (ii) of Lemma 4.3 we obtain

$$
\begin{aligned}
\mid-c^{-3} & \int_{|y-x| \leq c t} \int \tilde{a}^{\phi_{x_{i}}}(\omega, p) f\left(t-c^{-1}|y-x|, y, p\right) d p \frac{d y}{|y-x|^{2}} \mid \\
& \leq c^{-3} \int_{|y| \leq R+\mathcal{P}_{c}(t) t} \int_{|p| \leq \mathcal{P}_{c}(t)}\left|\tilde{a}^{\phi_{x_{i}}}(\omega, p)\right| f\left(t-c^{-1}|y-x|, y, p\right) d p \frac{d y}{|y-x|^{2}} \\
& \lesssim c^{-3} \sup _{0 \leq \tau \leq t}\|f(\tau)\|_{\infty} \mathcal{P}_{c}(t)^{5} \int_{|y| \leq R+t \mathcal{P}_{c}(t)} \int_{|p| \leq \mathcal{P}_{c}(t)} d p \frac{d y}{|y-x|^{2}} \\
& \lesssim c^{-3} \mathcal{P}_{c}(t)^{9}(1+t) e^{4 D t} .
\end{aligned}
$$

A computation similar to (4.3) shows that

$$
\left|1-\frac{1}{\left(1+c^{-1} \omega \cdot \widehat{p}\right)^{2} \sqrt{1+c^{-2} p^{2}}}\right| \lesssim c^{-1} \mathcal{P}_{c}(t)^{5} .
$$


Hence

$$
\begin{aligned}
& \mid c^{-2} \int_{|y-x| \leq c t} \int \omega_{i} f\left(t-c^{-1}|y-x|, y, p\right) d p \frac{d y}{|y-x|^{2}} \\
& -c^{-2} \int_{|y-x| \leq c t} \int \frac{\omega_{i}}{\left(1+c^{-1} \omega \cdot \widehat{p}\right)^{2} \sqrt{1+c^{-2} p^{2}}} f\left(t-c^{-1}|y-x|, y, p\right) d p \frac{d y}{|y-x|^{2} \mid} \mid \\
& \quad \lesssim c^{-2} \int_{|y| \leq R+\mathcal{P}_{c}(t) t} \int_{|p| \leq \mathcal{P}_{c}(t)} c^{-1} \mathcal{P}_{c}(t)^{5}\left|\omega_{i}\right| f\left(t-c^{-1}|y-x|, y, p\right) d p \frac{d y}{|y-x|^{2}} \\
& \quad \lesssim c^{-3} \mathcal{P}_{c}(t)^{9}(1+t) e^{4 D t} .
\end{aligned}
$$

Combining the estimates obtained thus far we get

$$
\begin{aligned}
& \left|\partial_{x_{i}} \phi_{\text {hom }}(t, x)-c^{-3} t^{-1} \int_{|y-x|=c t} \int \frac{\omega_{i}}{\left(1+c^{-1} \omega \cdot \widehat{p}\right) \sqrt{1+c^{-2} p^{2}}} f^{\text {in }}(y, p) d p d S_{y}+I_{x_{i}}\right| \\
& \lesssim\left|-c^{-2} \iint \omega_{i} f\left(\max \left\{0, t-c^{-1}|y-x|\right\}, y, p\right) d p \frac{d y}{|y-x|^{2}}\right|+c^{-3} \mathcal{P}_{c}(t)^{9} e^{4 D t} \\
& \lesssim c^{-2} \mathcal{P}_{c}(t)^{3} \sup _{[0, t]}\|f(s)\|_{\infty} \int_{|y| \leq R+\mathcal{P}_{c}(t) t} \frac{d y}{|y|^{2}}+c^{-3} \mathcal{P}_{c}(t)^{9} e^{4 D t} \\
& \lesssim c^{-2} \mathcal{P}_{c}(t)^{9}(1+t) e^{4 D t} .
\end{aligned}
$$

Now we estimate $I I_{x_{i}}$ and $I I I_{x_{i}}$. Again by (i) of Lemma 4.3 we obtain the bounds

$$
\left|b^{\phi_{x_{i}}}(\omega, p)\right| \lesssim \mathcal{P}_{c}(t)^{4}, \quad\left|c^{\phi_{x_{i}}}(\omega, p)\right| \lesssim \mathcal{P}_{c}(t)^{4}
$$

Let us define

$$
K_{c}(t)=\sup \left\{c\left|\partial_{t} \phi(t, x)\right|+c^{2}\left|\nabla_{x} \phi(t, x)\right|, x \in \mathbb{R}^{3}\right\} .
$$

Hence, using $c|S(\phi)| \leq K_{c}(t)$, we get

$$
\begin{aligned}
\left|I I_{x_{i} \mid}\right| & \lesssim c^{-3} \mathcal{P}_{c}(t)^{4} \int_{0}^{t} \int_{|y-x|=c(t-\tau)} \int_{|p| \leq \mathcal{P}_{c}(\tau)} f(\tau, y, p)|y-x|^{-1} K_{c}(\tau) d p d S_{y} d \tau \\
& \lesssim c^{-2} \mathcal{P}_{c}(t)^{7} t e^{4 D t} \int_{0}^{t} K_{c}(\tau) d \tau
\end{aligned}
$$

$I I I_{x_{i}}$ satisfies an identical estimate, since $c^{2}\left|\nabla_{x} \phi\right| \leq K_{c}(t)$. Collecting the various bounds we obtain

$$
\left|\nabla_{x} \phi(t, x)\right| \lesssim c^{-2} \mathcal{P}_{c}(t)^{9}(1+t) e^{4 D t}\left(1+\int_{0}^{t} K_{c}(\tau) d \tau\right)
$$

Now the estimate on the time derivative of the field. From Proposition 3.3 we have

$$
\begin{aligned}
\partial_{t} \phi(t, x)= & \partial_{t} \phi_{\mathrm{hom}}(t, x)-c^{-2} t^{-1} \int_{|y-x|=c t} \int \frac{f^{\mathrm{in}}(y, p)}{\left(1+c^{-1} \omega \cdot \widehat{p}\right) \sqrt{1+c^{-2} p^{2}}} d p d S_{y} \\
& +I_{t}+I I_{t}+I I I_{t},
\end{aligned}
$$


where

$$
\partial_{t} \phi_{\text {hom }}(t, x)=\frac{1}{c}\left[\partial_{t}\left(\frac{c^{-1} t}{4 \pi} \int_{|\omega|=1} h^{\sharp}(x+c t \omega) d \omega\right)+\frac{c t}{4 \pi} \int_{|\omega|=1} \Delta g^{\sharp}(x+c t \omega) d \omega\right] .
$$

A direct application of Lemma 4.1 now gives $\left\|\partial_{t} \phi_{\text {hom }}(t)\right\|_{\infty} \lesssim c^{-1}(1+t)$. For the second term of (4.6) we have

$$
\begin{aligned}
& c^{-2} t^{-1} \int_{|y-x|=c t} \int \frac{f^{\text {in }}(y, p)}{\left(1+c^{-1} \omega \cdot \widehat{p}\right) \sqrt{1+c^{-2} p^{2}}} d p d S_{y} \\
& \lesssim c^{-1} \mathcal{P}_{c}(0)^{2}\left[c t \int_{|\omega|=1} \int_{|p| \leq \mathcal{P}_{c}(0)} f^{\text {in }(x+c t \omega, p) d p d \omega] \lesssim c^{-1} .}\right.
\end{aligned}
$$

In order to estimate the remaining terms in (4.6) we use the bounds

$$
\left|a^{\phi_{t}}(\omega, p)\right| \lesssim \mathcal{P}_{c}(t)^{5}, \quad\left|b^{\phi_{t}}(\omega, p)\right| \lesssim \mathcal{P}_{c}(t)^{4}, \quad\left|c^{\phi_{t}}(\omega, p)\right| \lesssim \mathcal{P}_{c}(t)^{4} .
$$

The estimate for $I_{t}$ follows:

$$
\begin{aligned}
\left|I_{t}\right| & \lesssim c^{-2} \mathcal{P}_{c}(t)^{5} \int_{|y-x| \leq c t} \int_{|p| \leq \mathcal{P}_{c}(t)} f\left(t-c^{-1}|y-x|, y, p\right) d p \frac{d y}{|y-x|^{2}} \\
& \lesssim c^{-2} \mathcal{P}_{c}(t)^{8} \sup _{[0, t]}\|f(s)\|_{\infty} \int_{|y| \leq R+\mathcal{P}_{c}(t) t} \frac{d y}{|y|^{2}} \lesssim c^{-2} \mathcal{P}_{c}(t)^{9}(1+t) e^{4 D t}
\end{aligned}
$$

Also

$$
\begin{aligned}
\left|I I_{t}\right| & \lesssim c^{-2} \mathcal{P}_{c}(t)^{4} \int_{0}^{t} \int_{|y-x|=c(t-\tau)} \int_{|p| \leq \mathcal{P}_{c}(\tau)} f(\tau, y, p)|y-x|^{-1} K_{c}(\tau) d p d S_{y} d \tau \\
& \lesssim c^{-1} \mathcal{P}_{c}(t)^{7} t e^{4 D t} \int_{0}^{t} K_{c}(\tau) d \tau .
\end{aligned}
$$

$I I I_{t}$ satisfies an identical estimate, since $c^{2}\left|\nabla_{x} \phi\right| \leq K_{c}(t)$. Collecting the various bounds we obtain

$$
\left|\partial_{t} \phi(t, x)\right| \lesssim c^{-1} \mathcal{P}_{c}(t)^{9}(1+t) e^{4 D t}\left(1+\int_{0}^{t} K_{c}(\tau) d \tau\right) .
$$

Combining (4.7) and (4.5) entails

$$
K_{c}(t) \lesssim \mathcal{P}_{c}(t)^{9}(1+t) e^{4 D t}\left(1+\int_{0}^{t} K_{c}(\tau) d \tau\right)
$$

Hence by Gronwall's inequality,

$$
K_{c}(t) \lesssim \mathcal{P}_{c}(t)^{9}(1+t) e^{4 D t} \exp \left(\mathcal{P}_{c}(t)^{9}(1+t)^{2} e^{4 D t}\right)
$$

Note that the characteristics $(X, P)(s)$ of $(1.4)$ with $(X, P)(t)=(x, p)$ satisfies

$$
\frac{d P}{d s}=-(S \phi)(s, X) P-\frac{c^{2} \nabla_{x} \phi(s, X)}{\sqrt{1+P^{2}}} .
$$


So

$$
|p| \lesssim|P(0)|+\int_{0}^{t} \mathcal{K}_{c}(\tau) \mathcal{P}_{c}(\tau) d \tau \lesssim \mathcal{P}_{c}(0)-1+\int_{0}^{t} \mathcal{K}_{c}(\tau) \mathcal{P}_{c}(\tau) d \tau
$$

Therefore by (4.8) and the definition of $\mathcal{P}_{c}(t),(4.9)$ becomes

$$
\mathcal{P}_{c}(t) \lesssim 1+\int_{0}^{t}(1+\tau) \mathcal{P}_{c}(\tau)^{10} e^{4 D \tau} \exp \left(\mathcal{P}_{c}(\tau)^{9}(1+\tau)^{2} e^{4 D \tau}\right) d \tau .
$$

By Gronwall's inequality, there exists an interval $[0, T)$ independent of $c$ where $\mathcal{P}_{c}(t)$ remains finite for all $c \geq 1$, i.e., $\mathcal{P}_{c}(t) \lesssim 1$. Using this estimate we can complete the proof of Theorem 2.2. Let $T_{\max }^{c}$ denote the maximal time of existence of a solution of (1.3)-(1.4) and assume $T_{\max }^{c}<T$ for some $c \geq 1$. Since $\mathcal{P}_{c}(t)$ is an increasing function of time, this implies $\mathcal{P}_{c}\left(T_{\max }^{c}\right)<\infty$ and so, by Theorem $2.1, T_{\max }^{c}=\infty$, a contradiction. Hence, the solution is defined on the interval $[0, T)$ for all $c \geq 1$.

Proof of Theorem 2.3: For brevity we omit stating that the estimates below are valid for $t \in\left[0, T^{\prime}\right]$. The claim on $\partial_{t} \phi$ follows directly by (4.7) and the assumption $\mathcal{P}_{c}(t) \lesssim 1$. Note also that, by $(4.5), \nabla_{x} \phi=\mathcal{O}\left(c^{-2}\right)$. We start with estimating $\phi$. By the assumption we made on $\phi_{1}^{\text {in }}$ and Lemma 4.1, the second term in (3.2) becomes

$$
\frac{t}{4 \pi} \int_{|\omega|=1} \phi_{1}^{\mathrm{in}}(x+c t \omega) d \omega=\mathcal{O}\left(c^{-3}\right) .
$$

For the first term in (3.2), with the assumption on $\phi_{0}^{\text {in }}$, Lemma 4.2 and the fact that $\Delta g^{\sharp}=4 \pi \int f^{\text {in }} d p$, we get

$$
\begin{aligned}
\partial_{t}\left(\frac{t}{4 \pi} \int_{|\omega|=1} \phi_{0}^{\text {in }}(x+c t \omega) d \omega\right) & =-(4 \pi)^{-1} c^{-2} \int_{|y-x|>c t} \frac{\Delta g^{\sharp}(y)}{|y-x|} d y \\
& =-c^{-2} \int_{|y-x|>c t} \int \frac{f^{\text {in }}(y, p)}{|y-x|} d p d y .
\end{aligned}
$$

For $\psi(t, x)$ in (3.1), first consider

$$
\begin{aligned}
& \left|c^{-2} \int_{|y-x| \leq c t} \int \frac{f\left(t-c^{-1}|y-x|, y, p\right)}{|y-x|}-\frac{f\left(t-c^{-1}|y-x|, y, p\right)}{\sqrt{1+c^{-2} p^{2}}|y-x|} d p d y\right| \\
& \quad \lesssim c^{-2} \int_{|y| \leq R+\mathcal{P}_{c}(t) t} \int_{|p| \leq \mathcal{P}_{c}(t)} c^{-2} f\left(t-c^{-1}|y-x|, y, p\right) d p \frac{d y}{|y-x|} \lesssim c^{-4} .
\end{aligned}
$$

Then $\psi$ becomes

$$
\psi(t, x)=-c^{-2} \int_{|y-x| \leq c t} \int f\left(t-c^{-1}|y-x|, y, p\right) d p \frac{d y}{|y-x|}+\mathcal{O}\left(c^{-4}\right) .
$$

So collecting (4.11), (4.12) and (4.13), we get

$$
\begin{aligned}
\phi(t, x)=- & c^{-2} \int_{|y-x| \leq c t} \int f\left(t-c^{-1}|y-x|, y, p\right) d p \frac{d y}{|y-x|} \\
& -c^{-2} \int_{|y-x|>c t} \int \frac{f^{\text {in }}(y, p)}{|y-x|} d p d y+\mathcal{O}\left(c^{-3}\right) \\
= & -c^{-2} \iint f\left(\max \left\{0, t-c^{-1}|y-x|\right\}, y, p\right) d p \frac{d y}{|y-x|}+\mathcal{O}\left(c^{-3}\right) .
\end{aligned}
$$


So using (2.8) and (4.14), now we estimate

$$
\begin{aligned}
&\left|c^{2} \phi(t, x)-U(t, x)\right|= \mathcal{O}\left(c^{-1}\right)+\iint f_{\infty}(t, y, p) d p \frac{d y}{|y-x|} \\
&-\iint f\left(\max \left\{0, t-c^{-1}|y-x|\right\}, y, p\right) d p \frac{d y}{|y-x|} \mid \\
& \lesssim \iint\left|f_{\infty}\left(\max \left\{0, t-c^{-1}|y-x|\right\}, y, p\right)-f_{\infty}(t, y, p)\right| d p \frac{d y}{|y-x|} \\
& \quad+\iint \mid f\left(\max \left\{0, t-c^{-1}|y-x|\right\}, y, p\right) \\
& \quad-f_{\infty}\left(\max \left\{0, t-c^{-1}|y-x|\right\}, y, p\right) \mid d p \frac{d y}{|y-x|}+c^{-1}
\end{aligned}
$$

Define

$$
D_{F}(t):=\sup \left\{\left|f(\tau, x, p)-f_{\infty}(\tau, x, p)\right|: \tau \in[0, t], x \in \mathbb{R}^{3} \text { and } p \in \mathbb{R}^{3}\right\} .
$$

Also define $\mathcal{P}_{\infty}(t)$ as the following

$$
\mathcal{P}_{\infty}(t)=\sup _{0 \leq s<t}\left\{|p|:(x, p) \in \operatorname{supp} f_{\infty}(s)\right\}+1 .
$$

Since $\left(f_{\infty}, U\right)$ is a $C^{1}$ solution of (2.7)-(2.9) and the initial data $f_{\infty}$ has compact support, $\mathcal{P}_{\infty}$ is well defined for all $t \geq 0$. Note that $\partial_{t} f_{\infty}$ is bounded on $\mathbb{R}^{6} \times\left[0, T^{\prime}\right]$. Also let $\mathcal{P}(t):=\mathcal{P}_{c}(t)+\mathcal{P}_{\infty}(t)$. Then (4.15) becomes

$$
\begin{aligned}
& \left|c^{2} \phi(t, x)-U(t, x)\right| \\
& \quad \lesssim \int_{|y| \leq R+\mathcal{P}(t) t} \int_{|p| \leq \mathcal{P}(t)} \int_{\max \left\{0, t-c^{-1}|y-x|\right\}}^{t}\left|\partial_{t} f_{\infty}(s, y, p)\right| d s d p \frac{d y}{|y-x|} \\
& \quad+\int_{|y| \leq R+\mathcal{P}(t) t} \int_{|p| \leq \mathcal{P}(t)} D_{F}\left(\max \left\{0, t-c^{-1}|y-x|\right\}\right) d p \frac{d y}{|y-x|}+c^{-1} . \\
& \quad \lesssim D_{F}(t)+c^{-1} .
\end{aligned}
$$

Before estimating $D_{F}(t)$, let us look at $\nabla_{x} \phi$. In the process of proving Theorem 2.2 we have also shown that

$$
\begin{gathered}
\partial_{x_{i}} \phi_{\mathrm{hom}}-c^{-3} t^{-1} \int_{|y-x|=c t} \int \frac{\omega_{i}}{\left(1+c^{-1} \omega \cdot \widehat{p}\right) \sqrt{1+c^{-2} p^{2}}} f^{\mathrm{in}}(y, p) d p d S_{y}+I_{x_{i}} \\
=-c^{-2} \iint \omega_{i} f\left(\max \left\{0, t-c^{-1}|y-x|\right\}, y, p\right) d p \frac{d y}{|y-x|^{2}}+\mathcal{O}\left(c^{-3}\right), \quad
\end{gathered}
$$

cf. (4.4). For $I I_{x_{i}}$ in (4.1) we use $S(\phi)=\mathcal{O}\left(c^{-1}\right)$ and $\left|b^{\phi_{x_{i}}}\right| \lesssim \mathcal{P}_{c}(t)^{4}$. Therefore

$$
\left|I I_{x_{i}}\right| \lesssim c^{-3} \int_{|y| \leq R+\mathcal{P}_{c}(t) t} \int_{|p| \leq \mathcal{P}_{c}(t)} c^{-1} f\left(t-c^{-1}|y-x|, y, p\right) d p \frac{d y}{|y-x|} \lesssim c^{-4}
$$

The estimation of $I I I_{x_{i}}$ is similar to the one of $I I_{x_{i}}$. Recall that $\left|c^{\phi_{x_{i}}}\right| \lesssim \mathcal{P}_{c}(t)^{4}$ and $\nabla_{x} \phi=\mathcal{O}\left(c^{-2}\right)$. So we get

$$
\left|I I I_{x_{i}}\right| \lesssim c^{-4}
$$


Now collecting (4.17)-(4.19) we obtain

$$
\partial_{x_{i}} \phi=-c^{-2} \iint\left(y_{i}-x_{i}\right) f\left(\max \left\{0, t-c^{-1}|y-x|\right\}, y, p\right) d p \frac{d y}{|y-x|^{3}}+\mathcal{O}\left(c^{-3}\right) .
$$

By the similar argument in (4.16), with (2.8) and (4.20), we estimate

$$
\begin{aligned}
\left|c^{2} \nabla_{x} \phi(t, x)-\nabla_{x} U(t, x)\right| & \\
=\mid & \mathcal{O}\left(c^{-1}\right)+\iint(y-x) f_{\infty}(t, y, p) d p \frac{d y}{|y-x|^{3}} \\
& \quad-\iint(y-x) f\left(\max \left\{0, t-c^{-1}|y-x|\right\}, y, p\right) d p \frac{d y}{|y-x|^{3} \mid} \mid \\
\lesssim & \int_{|y| \leq R+\mathcal{P}(t) t} \int_{|p| \leq \mathcal{P}(t)} \int_{\max \left\{0, t-c^{-1}|y-x|\right\}}^{t}\left|\partial_{t} f_{\infty}(s, y, p)\right| d s d p \frac{d y}{|y-x|^{2}} \\
\quad & \quad \int_{|y| \leq R+\mathcal{P}(t) t} \int_{|p| \leq \mathcal{P}(t)} D_{F}\left(\max \left\{0, t-c^{-1}|y-x|\right\}\right) d p \frac{d y}{|y-x|^{2}}+c^{-1} \\
\lesssim & D_{F}(t)+c^{-1} .
\end{aligned}
$$

To estimate $D_{F}(t)$, let us define $D_{f}:=f-f_{\infty}$. Then using the two Vlasov equations (1.4) and (2.7), we obtain

$$
\begin{aligned}
\partial_{t} & D_{f}+\widehat{p} \cdot \nabla_{x} D_{f}-\left[S(\phi) p+\frac{c^{2} \nabla_{x} \phi}{\sqrt{1+c^{-2} p^{2}}}\right] \cdot \nabla_{p} D_{f} \\
& =(p-\hat{p}) \cdot \nabla_{x} f_{\infty}+\left[S(\phi) p+\frac{c^{2} \nabla_{x} \phi}{\sqrt{1+c^{-2} p^{2}}}-\nabla_{x} U\right] \cdot \nabla_{p} f_{\infty}+4 S(\phi)\left(f_{\infty}+D_{f}\right) .
\end{aligned}
$$

Note that $|p-\widehat{p}| \leq c^{-2} \mathcal{P}_{c}(t)^{3}$. Also note that $\nabla_{x} f_{\infty}, \nabla_{p} f_{\infty}$ and $f_{\infty}$ are bounded on $\mathbb{R}^{6} \times\left[0, T^{\prime}\right]$. Then with (4.21), (4.22) becomes

$$
\begin{gathered}
\left|\partial_{t} D_{f}+\widehat{p} \cdot \nabla_{x} D_{f}-\left[S(\phi) p+\frac{c^{2} \nabla_{x} \phi}{\sqrt{1+c^{-2} p^{2}}}\right] \cdot \nabla_{p} D_{f}\right| \\
\lesssim c^{-1}+\left|c^{2} \nabla_{x} \phi-\nabla_{x} U\right|+c^{-1}\left|D_{f}\right| \lesssim D_{F}(t)+c^{-1} .
\end{gathered}
$$

Using the characteristics $(X, P)(s)$ of $(1.4)$ with $(X, P)(t)=(x, p)$, compute

$$
\left|\frac{d}{d s} D_{f}(s, X(s), P(s))\right| \lesssim D_{F}(s)+c^{-1}
$$

Note that $D_{f}(0, X(0), P(0))=0$. Therefore integrating (4.23) we get

$$
D_{F}(t) \lesssim \int_{0}^{t} D_{F}(s) d s+c^{-1}
$$

So Gronwall's inequality implies $D_{F}(t) \lesssim c^{-1}$, which gives (2.14). The proof of (2.13) is completed by (4.16) and (4.21). This concludes the proof of Theorem 2.3.

Acknowledgments. The authors thank Gerhard Rein and Alan D. Rendall for comments on the manuscript and suggestions. S. C. acknowledges the Albert Einstein Institute for the kind hospitality and the European network HYKE for financial support (contract HPRN-CT-2002-00282). 


\section{REFERENCES}

[1] C. Brans and R.H. Dicke, Mach's principle and a relativistic theory of gravitation, Phys. Rev., 124(3):925-935, 1961.

[2] S. Calogero, Spherically symmetric steady states of galactic dynamics in scalar gravity, Class. Quant. Gravity, 20:1729-1741, 2003.

[3] S. Calogero, Global small solutions of the Vlasov-Maxwell system in the absence of incoming radiation, Indiana Univ. Math. Journal (to appear), preprint: math-ph/0211013.

[4] S. Calogero and G. Rein, On classical solutions of the Nordström-Vlasov system, Commun. Partial Diff. Eqns., 28:1863-1885, 2003.

[5] S. Calogero and G. Rein, Global weak solutions to the Nordström-Vlasov system, preprint: math-ph/0309046.

[6] T. Damour, G. Esposito-Farese, Tensor-multi-scalar theories of gravitation, Class. Quant. Gravity, 9:2093-2176, 1992.

[7] R. DiPerna and P.L. Lions, Global weak solutions of Vlasov-Maxwell systems, Comm. Pure Appl. Math., 42(6):729-757, 1989.

[8] A. Einstein and A.D. Fokker, Die Nordströmsche gravitationstheorie vom standpunkt des absoluten Differentialkalküls, Annalen der Physik, 44:321-328, 1914.

[9] R. Glassey and J. Schaeffer, Global existence for the relativistic Vlasov-Maxwell system with nearly neutral initial data, Comm. Math. Phys., 119:353-384, 1988.

[10] R. Glassey and J. Schaeffer, The "two and one-half dimensional" relativistic Vlasov-Maxwell system, Comm. Math. Phys., 185:257-284, 1997.

[11] R. Glassey and J. Schaeffer, The relativistic Vlasov Maxwell system in two space dimensions, Part I. Arch, Rational Mech. Anal., 141:331-354, 1998.

[12] R. Glassey and J. Schaeffer, The relativistic Vlasov Maxwell system in two space dimensions, Part II. Arch, Rational Mech. Anal., 141:335-374, 1998.

[13] R. Glassey and W. Strauss, Singularity formation in a collisionless plasma could occur only at high velocities, Arch. Rat. Mech. Anal., 92:59-90, 1986.

[14] R. Glassey and W. Strauss, Absence of shocks in an initially dilute collisionless plasma, Comm. Math. Phys., 113:191-208, 1987.

[15] S. Klainerman and G. Staffilani, A new approach to study the Vlasov-Maxwell system, Comm. Pure Appl. Anal., 1:103-125, 2002.

[16] H. Lee, The classical limit of the relativistic Vlasov-Maxwell system in two space dimensions, Math. Meth. Appl. Sci., (to appear).

[17] P.L. Lions and B. Perthame, Propagation of moments and regularity for the 3-dimensional Vlasov-Poisson system, Invent. Math., 105:415-430, 1991.

[18] G. Nordström, Zur Theorie der Gravitation vom Standpunkt des Relativitätsprinzips, Ann. Phys. Lpz., 42:533, 1913.

[19] K. Pfaffelmoser, Global classical solutions of the Vlasov-Poisson system in three dimensions for general initial data, J. Diff. Eqns., 95:281-303, 1992.

[20] G. Rein, Selfgravitating systems in Newtonian theory - the Vlasov-Poisson system, Banach Center Publications 41 (Part I):179-194, 1997.

[21] G. Rein, Generic global solutions of the relativistic Vlasov-Maxwell system of plasma physics, Comm. Math. Phys., 135:41-78, 1990.

[22] G. Rein and A.D. Rendall, Global existence of solutions of the spherically symmetric VlasovEinstein system with small initial data, Commun. Math. Phys., 150:561-583, 1992.

[23] G. Rein and A.D. Rendall, The Newtonian limit of the spherically symmetric Vlasov-Einstein system, Commun. Math. Phys., 150:585-591, 1992.

[24] A.D. Rendall, The Newtonian limit for asymptotically flat solutions of the Vlasov-Einstein system, Commun. Math. Phys., 163:89-112, 1994

[25] J. Schaeffer, The classical limit of the relativistic Vlasov-Maxwell system, Comm. Math. Phys., 104:409-421, 1986

[26] J. Schaeffer, Global existence of smooth solutions to the Vlasov-Poisson system in three dimensions, Commun. Part. Diff. Eqns., 16:1313-1335, 1991. 